\title{
Flushing Disorders Associated with Gastrointestinal Symptoms: Part 2, Systemic Miscellaneous Conditions
}

\author{
Vaibhav Rastogi, MD; Devina Singh, MD; Joseph J. Mazza, MD; \\ Dipendra Parajuli, MD, and Steven H. Yale, MD
}

\begin{abstract}
Flushing disorders with involvement of the gastrointestinal tract represent a heterogeneous group of conditions. In part I of this review series, neuroendocrine tumors (NET), mast cell activation disorders (MCAD), and hyperbasophilia were discussed. In this section we discuss the remaining flushing disorders which primarily or secondarily involve the gastrointestinal tract. This includes dumping syndrome, mesenteric traction syndrome, rosacea, hyperthyroidism and thyroid storm, anaphylaxis, panic disorders, paroxysmal extreme pain disorder, and food, alcohol and medications. With the exception of paroxysmal pain disorders, panic disorders and some medications, these disorders presents with dry flushing. A detailed and comprehensive family, social, medical and surgical history, as well as recognizing the presence of other systemic symptoms are important in distinguishing the different disease that cause flushing with gastrointestinal symptoms.
\end{abstract}

Keywords: Flushing; Gastrointestinal; Human; Panic disorder; Dumping syndrome

$\mathrm{N}$ euroendocrine tumors, mast cell disorders, and hyperbasophilia are diseases that arise from the gastrointestinal tract or causes gastrointestinal symptoms and were covered in part 1 of this review. These diseases vary based on their malignant potential, and all cause dry flushing, with diagnosis based on biochemical properties and in some cases histopathology obtained from tissue and/or bone marrow biopsy.

In Part 2 of this review, we cover common and rare causes of flushing, including dumping syndrome, mesenteric traction syndrome, rosacea, hyperthyroidism and thyroid storm, anaphylaxis, panic disorders, paroxysmal extreme pain disorder, and food, alcohol and medications. These are a heterogeneous group of disease that share similar nonspecific gastrointestinal symptoms including nausea, diarrhea and abdominal pain (Figure 1). These conditions are differentiated based on a comprehensive history that should include duration, frequency, and factors that triggers symptoms since in some of these diseases there are no biomarkers to confirm the disease.

\section{Methods}

A description of the methodological approach to this review can be found in Part 1, Neuroendocrine Tumors, Mast Cell Disorders, and Hyperbasophilia.

\section{Dumping Syndrome}

Dumping syndrome is a condition characterized by rapid emptying of the contents of the stomach into the small intestines postprandially. It occurs post-operatively after esophageal, bariatric, and gastric surgery, and in patients with diabetes mellitus. In some patients the condition is idiopathic. ${ }^{1}$ This syndrome occurs in approximately $20 \%$ of patients
Corresponding Author: Steven H. Yale MD, University of Central Florida College of Medicine, HCA Consortium Graduate Medical Education, North Florida Regional Medical Center, 6500 W Newberry Rd, Gainesville, FL 32605, Tel: (7I5) 383-0928, Email: steven.yale.md@gmail.com
Received: July 31, 2017

Revised: November 30, 2017

Accepted: December 21, 2017

doi: $10.3121 / \mathrm{cmr} .2017 .1379 \mathrm{~b}$ 
Flushing with GI symptoms

- Review for food, alcohol, medication, poisoning, occupation and if present remove exposure.

- Hyperacute onset with angioedeman evaluate for anaplylaxis.

- If neither of these findings are present evaluate based on the following symptoms:

$\cdot$ Headache, tachycardia, sweating, hypertension $\rightarrow$ pheochromocytomas, ganglioneuroma

-Bronchospasm, gastrointestinal bleeding, obstruction, vasoconstriction $\rightarrow$ NETs

-Vasodilation, hypotension, tachycardia $\rightarrow$ Mast cell activation disorders

•Polyneuropathy, lymphadenopathy, endocrine symptoms $\rightarrow$ POEMS Syndrome

$\cdot$ Hypokalemia, achlorhydria $\rightarrow$ VIPoma

-Palpitation., tremor, fatigue, hair thinning $\rightarrow$ Hyperthyroidism

•Post gastric by-pass/ bariatric surgery with post-prandial rapid gastric emptying $\rightarrow$ Dumping syndrome

-Recent abdominal surgery $\rightarrow$ Mesenteric traction syndrome

-Dysphagia, dyspnea $\rightarrow$ Medullary carcinoma of thyroid

-Visceral pain paroxysm with family history $\rightarrow$ Paroxysmal extreme pain disorder

-Behavioral/ emotional/ mood disorders $\rightarrow$ Panic disorder

-Dysesthesia and cutaneous findings $\rightarrow$ Rosacea and other dermatological disorders

-Basophilic count $>1000$ per ul blood $\rightarrow$ Hyperbasophic disorders

\section{Flushing without GI symptoms}

- Menopause

- Medications (e.g. GnRH)

- Physiological (heat, anger, embarrassment, drinks)

- Auriculotemporal syndrome (Frey's Syndrome)

- Harlequin syndrome

- Pseudocarcinoid syndrome in secondary male hypogonadism

- Spinal cord injury above T6 (autonomic hyperreflexia)

- Rapid increase in intracranial pressure

- Migraine headaches

- Horseshoe kidney (Rovsing's syndrome)

- Renal cell carcinoma

Figure 1. Diagnostic approach to flushing with and without gastrointestinal symptoms.

undergoing pylorotomy and vagotomy, 40\% post-roux-en-y gastric bypass or sleeve gastrectomy, and 50\% after esophagectomy procedures. ${ }^{2}$ Pathogenesis is due to alterations in gastric anatomy or its innervation mediated by various gastrointestinal peptides such as vasoactive intestinal polypeptide, neurotensin, and incretins..$^{2,3}$

\section{Clinical Presentation and Gastrointestinal Involvement}

Early and late variants of dumping syndrome have been described depending upon the timing of symptom onset (within 1 hour or up to 3 hours) postprandially. ${ }^{2}$ Symptoms caused by the rapid hyperosmolar load transmitted from the stomach to the small intestines consist of abdominal cramps, diarrhea, nausea, vomiting, and postprandial fatigue. Flushing, palpitations, perspiration, hypotension, tachycardia and headache also occur with elevated bradykinin levels implicated in causing flushing. ${ }^{1}$

\section{Diagnosis and Treatment}

Glucose challenge test and gastric emptying scintigraphy are diagnostic. Non-pharmacologic treatments include consuming smaller and more frequent meals supplemented with fiber and protein $^{1}$ and medications such as acarbose, octreotide, diazoxide, loperamide, and nifedipine. ${ }^{2}$ Surgical approaches or continuous enteral feeding should be considered in refractory disease. ${ }^{2}$

\section{Mesenteric Traction Syndrome}

Mesenteric traction syndrome (MTS) is a rare condition seen in the early phase of abdominal upper gastrointestinal, pancreatic, and abdominal vascular surgery. ${ }^{4}$ As the name suggests it occurs after eventeration and manipulation, and it was initially called eventeration syndrome. ${ }^{5}$

\section{Clinical Presentation and Gastrointestinal Involvement}

The triad of flushing, hypotension, and tachycardia characterizes MTS. Episodes last for approximately 30 minutes..$^{5}$ Mast cells and endothelial cells are primarily involved in the pathogenesis with prostacyclin (PGI2) release suggested to be the primary mediator of this syndrome. ${ }^{5}$ Other mediators involved include histamine, bradykinin, nitric oxide and serotonin.

\section{Diagnosis and Treatment}

Mesenteric traction syndrome is a clinical diagnosis. Cyclooxygenase inhibitors are used to treat or prevent the occurrence of this syndrome. ${ }^{4}$ Other classes of medications used include histamine-1 antagonists and serotonin receptor

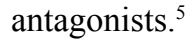

\section{Rosacea}

Rosacea is a chronic relapsing inflammatory and vascular skin disorder of unknown etiology. Prevalence ranges from $1 \%$ to $20 \%$ and typically occurs in patients after the third decade of life. ${ }^{6}$ It most commonly involves the central face and occurs principally in fair-skinned individuals. ${ }^{7}$ Genetic and environmental factors are believed to account for the pathogenesis of this disease. Risk factor for development of the disease includes family history, lighter phototypes, increased alcohol consumption, and excessive ultraviolet exposure. ${ }^{6}$ Of the four subtypes of rosacea established by the National Rosacea Society, flushing occurs in subtype 1 or 
erythmatotelangiectatic variant. ${ }^{6,89}$ Prolonged flushing and erythema with or without telangiectasias involves the face, ears, neck and upper trunk. Patients may also report edema, rough or scaly skin, edema and stinging or burning sensation suggestive of an antidromic autonomic response to vasodilation..$^{9,10}$

Flushing and increased blood flow is believed to be the result of vasodilatory neural stimuli and humoral release of a variety of mediators that includes the neuropeptides vascular endothelial growth factor (VEGF), vasoactive intestinal peptide (VIP), substance $\mathrm{P}$, and acetylcholine (Ach). ${ }^{9,11}$ Evidence for a neurogenic component is further suggested by the findings of upregulation of neuropeptide genes: VIP, pituitary adenylate cyclase-activating polypeptide (PACP), 5-hydyroxytryptmine $3 \mathrm{~A}$ receptors, nerve growth factor beta, alpha-1D adrenergic receptors, adrenomedullin 2, and cathelicidin antimicrobial peptide. ${ }^{11,12}$ Transient receptor potential vanilloid of cation channels and ankyrin receptors may also contribute to flushing and burning. ${ }^{13}$

\section{Clinical Presentation and Gastrointestinal Involvement}

Flushing and facial redness is a sign of early disease. Symptoms are recurrent lasting up to 3 hours. ${ }^{6}$ Some studies suggest an association between rosacea and gastrointestinal tract disorders including celiac disease, irritable bowel syndrome, inflammatory bowel disease, small intestinal overgrowth and Helicobacter pylori infection. ${ }^{7,14,15}$ The pathogenesis behind this relationship remains unknown.

\section{Diagnosis and Treatment}

Rosacea is diagnosed clinically based on characteristic features. ${ }^{7}$ Measures to prevent flushing involve avoiding triggers such as protection from ultraviolet rays from the sun, spicy food, hot beverages and alcohol. ${ }^{8}$ Pharmacologic management includes metronidazole, azelaic acid, ivermectin, or sulfur preparations. ${ }^{16}$ Erythema of the face can be managed with topical $\alpha$ adrenergic receptor agonists such as brimonidine tartrate gel $0.05 \% .{ }^{17}$ Progressive and severe disease is treated with oral antibiotics and retinoids, and laser procedures are used to treat telangiectasia and rhinophyma. ${ }^{16}$

\section{Hyperthyroidism and Thyroid Storm}

Hyperthyroidism, a condition caused by elevated thyroid hormone production, has a prevalence of $1.3 \%$ in United States. ${ }^{18}$ It is more common in females with the incidence increasing by age. Conditions that cause hyperthyroidism, including Grave's disease, Hashitoxicosis, toxic adenoma, multi-nodular goiter and thyroiditis, may cause flushing. ${ }^{18}$ Thyroid storm is a condition resulting in the acute increase in thyroid hormone levels. It can be precipitated by aberrant discontinuation or improper dosing of anti-thyroid medications, thyroid or non-thyroidal surgery, infection, trauma and parturition.

Clinical Presentation and Gastrointestinal Involvement Gastrointestinal manifestations related to excess of thyroid hormone include diarrhea, malabsorption, nausea, vomiting and abdominal pain caused by increased gut motility. Goiter can cause local esophageal compression and dysphagia. ${ }^{18}$ Cutaneous symptoms include warmth, flushing, sweating, pruritus, and thinning of the hair. Other constitutional and clinical manifestations include weight loss, tremors, palpitations, dyspnea, fatigue, sleep abnormalities and diplopia. $^{18}$

Additional symptoms that may occur in thyroid storm include tachycardia, agitation, hyperthermia, psychosis, severe dyspnea, diarrhea, nausea, vomiting, flushing, and hepatitis. ${ }^{19,20}$

\section{Diagnosis and Treatment}

Tests of thyroid function (thyroid stimulating hormone, free and total T4, free and total T3) are used to confirm the diagnoses. Treatment for hyperthyroidism involves antithyroid medication, surgery, and radioactive iodine therapy. ${ }^{18}$ Thyroid storm requires treatment with beta-blockers for symptomatic management and iodine and thioamide (methimazole) to prevent synthesis and release of thyroid hormone. Corticosteroids and iodinated radiocontrast agent block peripheral T4 to T3 conversion and have also been found useful in management. ${ }^{19,20}$

\section{Anaphylaxis}

Anaphylaxis is an acute potentially lethal condition involving multiple organ systems caused by the abrupt release of mediators from mast cell and/or basophils. ${ }^{21,22}$ Immunoglobulin $\mathrm{G}$ (IgG), IgE and complements play an important role in the pathophysiology of anaphylactic patients. ${ }^{22}$ Most cases are related to food, drugs (penicillins) and insect stings while onethird are idiopathic. Some patients may have a genetic predisposition such as in hereditary angioedema. ${ }^{22}$ The incidence of anaphylaxis in the United State is approximately $1 \%$ to $3 \% .^{22}$ Anaphylactoid reactions, which clinically resemble anaphylaxis, are often precipitated by non-IgE related mechanisms.

\section{Clinical Presentation and Gastrointestinal Involvement}

Cutaneous symptoms of flushing, angioedema, and urticaria are found in the majority of patients. ${ }^{23}$ Gastrointestinal manifestations including abdominal pain, nausea, vomiting and diarrhea. ${ }^{22}$ Severe symptoms may include shortness of breath, laryngospasm, coughing, congestion, choking, tachycardia, hypotension, syncope, and dizziness.

\section{Diagnosis and Treatment}

Diagnosis is primarily clinically based with the treatment of choice being emergent administration of intramuscular or intravenous epinephrine. ${ }^{21}$ Confirmatory markers, although not generally obtained, include finding an elevated tryptase level indicative of mast cell degranulation. In non-emergent cases, allergy or prick testing can be pursued to identify the culprit allergen. ${ }^{21}$ 


\section{Panic Disorder}

The American Psychiatric Association classifies panic disorder as an anxiety disorder in DSM-5 that is a clinical syndrome characterized by recurrent autonomic and dissociative clinical symptoms and intense irrational fear and discomfort of recurrence. Symptoms occur spontaneously or in response to a known trigger, peak within minutes and last for few minutes to an hour. ${ }^{24,25}$ Currently, lifetime presence of panic disorder in the United States in patients between the ages of 15 years and 54 years is approximately $4 \%$ to $7 \%$. It is believed that around $33 \%$ of people may have at least one panic attack at some time during their lives. ${ }^{26}$

\section{Clinical Presentation and Gastrointestinal Involvement}

Diagnoses requires the presence of a minimum of four of 13 symptoms including palpitations, chest pain, sweating, shaking, dizziness, flushing, stomach churning, numbness, choking feeling, hot or cold sensation, de-realization, breathlessness and fear of losing control or dying, along with concerns about maladaptive behavioral changes resulting in an attempt to avoid further episodes (Table 1). ${ }^{24,25}$ Flushing in panic disorder is of the wet type associated with activation of the autonomic nervous system and stimulation of eccrine sweat glands. ${ }^{27}$

Panic disorder may be linked to structural and functional gastrointestinal diseases including peptic ulcer disease, gastritis, irritable bowel syndrome and chest pain of esophageal origin. ${ }^{28-31}$ Studies suggest that cholecystokinin might be a common mediator between functional gastrointestinal diseases and panic disorder. ${ }^{32}$ Dysregulation within the locus coeruleus of the brainstem area in patients with panic disorder may be responsible for symptoms of irritable bowel syndrome due to interactions between the central and enteric nervous system. ${ }^{30}$

\section{Diagnosis and treatment}

Diagnosis is based on identifying at least four physical and psychological symptoms. Medical and psychotherapy alone or in combination, particularly in patients with moderate to severe disease, are effective in preventing and controlling symptoms. Medications used to treat panic disorders include anti-depressants, benzodiazepines, and selective serotonin reuptake inhibitors, with the latter considered as first line therapy. ${ }^{24}$

\section{Parosysmal Extreme Pain Disorder}

Paroxysmal Extreme Pain Disorder (PEPD), formerly named Familial Rectal Pain Syndrome, is a rare autosomal dominant clinical syndrome characterized in adults primarily by excruciating burning pain in the rectal, ocular and mandibular regions and lower body, autonomic (flushing, rhinorrhea, diaphoresis), cardiovascular (bradycardia, asystole, syncope), and tonic nonepileptic seizures. Symptoms occur in response to benign mechanical triggers such as defecation (rectal crisis), yawning and eating (mandibular crisis), spontaneously (ocular crisis) as well as cold temperatures and emotional factors. ${ }^{33-36}$

PEPD is caused by a heterozygous missense mutation (gain of function) in the SCN9A gene expressed in peripheral sensory nerves of the dorsal root and sympathetic ganglion neurons that encode the alpha-subunit of Nav1. ${ }^{7}$ voltage-gated sodium channel. This sodium channel is responsible for the generation and propagation of the action potential in primary afferent

Table 1. Four DSM-5 Criteria for Panic Disorder Based Flushing ${ }^{25}$

A. Four or more of the following symptoms occurring during a panic attack

1. Palpitations, pounding heart or accelerated heart rate.

2. Sweating

3. Trembling or shaking

4. Sensation of shortness of breath or smothering

5. Feeling of choking

6. Chest pain or discomfort

7. Nausea or abdominal distress

8. Feeling dizzy, unsteady, light-headed, or faint

9. Chills or heat sensations.

10. Paresthesias

11. Derealization or depersonalization

12. Fear of losing control

13. Fear of dying

B. At least one of the attacks has been followed by at least one month of one or both of the following:

1. Persistent concern or worry about additional panic attacks or their consequences

2. A significant maladaptive change in behavior related to the attack(s).

C. The disturbance is not attributable to the physiological effects of a substance or another medical condition.

D. The disturbance is not better explained by another mental disorder 
sensory and sympathetic autonomic nerve fibers. ${ }^{35}$ Alterations in closure of the channel leads to enhanced cellular depolarization allowing more channels to remain open and hyperexcitable at the defined membrane potential..$^{35,37}$

\section{Clinical Presentation and Gastrointestinal Involvement} PEPD is an autosomal dominant disease. Symptoms begin during the neonatal period and progress with increasing age. ${ }^{38}$ Patients and affected family members present with visceral pain paroxysms and diverse symptoms based on the severity, locations and duration of symptoms. ${ }^{34,35}$ Patient may initially complain of pruritus followed by burning pain that when severe is described as sharp, stabbing or lancinating, lasting for seconds to hours and gradually subsides with time. ${ }^{34}$ Although pain may initially start in a specific region, during severe attacks it may become more widespread and affect the entire body. ${ }^{34}$ Flushing and diaphoresis are mediated through dysfunction of autonomic nerves and typically occur at the pain site. Constipation is commonly reported between attacks. ${ }^{34}$ In contrast to ocular and mandibular crises, the frequency and severity of rectal crisis tends to decrease with advancing age. ${ }^{34}$

\section{Diagnosis and Treatment}

PEPD is a clinical diagnosis based on signs and symptoms that vary among affected individuals and family members. Genetic sequencing of SCN9A may assist in confirming the diagnosis. Medical therapy including amitriptyline, gabapentin, carbamazepine, topiramate, valproic acid and tiagabine has shown variable effectiveness in reducing the frequency and severity of attacks. ${ }^{38}$ Non-pharmacologic measures involve pelvic floor retraining with biofeedback. ${ }^{39}$

\section{Food, Alcohol and Medications}

Flushing and alterations in gastrointestinal motility occur with a variety of medications and chemotherapeutic agents including cholinergic agents, morphine, catecholamines, vancomycin, rifampicin, doxorubicin, selective serotonin reuptake inhibitors, tramadol and cyclosporine (Table 2). ${ }^{40,41}$ Gastrointestinal symptoms include diarrhea, abdominal pain, nausea and vomiting. The mechanism precipitating symptoms and the presence of other clinical features vary depending on the offending agent. ${ }^{21,42}$ Treatment involves symptomatic management, dose adjustment or discontinuation of the responsible medication. Pretreatment, in some cases, with anti-histaminergics can prevent the development of symptoms. $^{42}$

Alcohol, a vasodilator, is another agent that may cause flushing accompanied by nausea and vomiting particularly in susceptible individuals who lack the enzyme aldehyde dehydrogenase involved in alcohol metabolism. It is believed to be genetically determined and is most commonly found in the Asian population. ${ }^{43}$ Additionally, inhibition of aldehyde dehydrogenase by disulfiram or other disulfiram-like inducing agents such as chloramphenicol, furazolidone, metronidazole, cephaperazone, tolbutamide can cause flushing due to impaired metabolism and accumulation of acetaldehyde..$^{21,44}$ Abstinence from alcohol in those who lack aldehyde dehydrogenase or who are consuming medications that inhibit aldehyde dehydrogenase prevents symptoms. ${ }^{21}$

Improper or delayed refrigeration of primarily dark meat fish (mainly tuna from Scrombridae and Scromberescidae families) may cause symptoms consisting of flushing, sweating, headache, cramping abdominal pain, nausea, vomiting and diarrhea to occur shortly after consumption. ${ }^{45-47}$ Other fish of the non-scromboid family (eg, swordfish, spotted sardines) have also been reported to cause this syndrome. Histamine is the main mediator for the symptoms of scombroid fish poisoning and is caused by bacteria within the fish, which under warm conditions converts histidine to histamine by the enzyme histamine decarboxylase. Cooking kills the bacteria but histamine remains intact since it is heat stable. Symptoms generally resolve within 24 hours. Antihistaminic medications are used in symptom management. ${ }^{45,46}$

Ingestion of the inocybe mushroom has been reported to cause an acute syndrome of flushing, nausea, vomiting, diarrhea, abdominal pain and a variety of muscarinic manifestations including hypersalivation, diaphoresis, bradycardia, lacrimation, and blurred vision. ${ }^{48}$ Treatment is supportive and includes intravenous fluids, atropine and antiemetics. ${ }^{48}$

Sulphides used as a preservative and additive to food and drinks has also been reported to cause symptoms of flushing, abdominal pain, diarrhea, urticaria and hypotension in susceptible individuals. ${ }^{49}$ Preventive measures include avoiding food and beverage containing high concentration of sulphides such as grape, lemon or lime juice, wine and most dry fruits.

\section{Conclusion}

The differential diagnosis of flushing can be challenging, since it encompasses both benign and malignant conditions and includes a wide variety of overlapping non-specific gastrointestinal symptoms. Initial evaluation involves obtaining a detailed history and physical examination inquiring about the frequency and duration of the attacks, temporal factors, description and location, and precipitating factor(s). Food, beverages and alcohol are the most prevalent causes for flushing in combination with gastrointestinal symptoms (usually nausea, vomiting and diarrhea). Inquiry should be made into whether the patient experiences episodes of intense fears or discomfort associated with a variety of non-specific symptoms such as palpitations, trembling, sweating and chest pain suggestive of a panic disorder. The constellation of flushing, diarrhea and hypotension suggests mast cell activation syndromes, anaphylaxis, pheochromocytomas, scromboid poisoning, and carcinoid syndrome. Absence of sweating and pruritus with burning pain and flushing may be helpful in narrowing the differential diagnosis to a mast cell activation disorder. Asking the patient to maintain a diary for 2 weeks recording the time of occurrence and factor that triggered the 


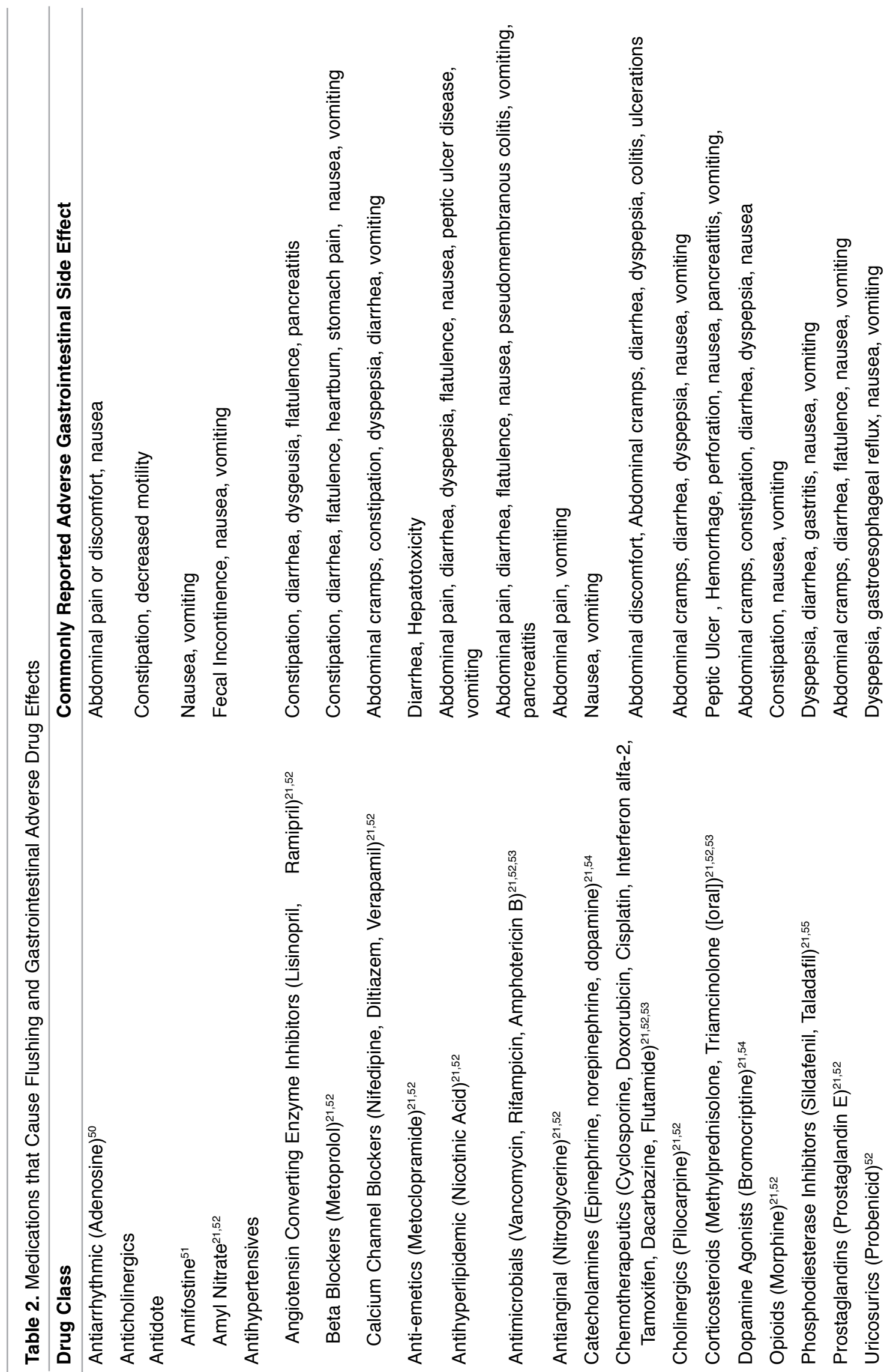

$34 \quad$ Flushing associated with gastrointestinal system 
flushing episode may be helpful in cases were the history is not clear or incomplete during initial assessment.

Recognizing the pattern of disease and key clinical features through the history provides important clues in initial assessment. Obtaining specific biochemical tests is based on pre-test probability of disease. Radiologic imaging, if required, should be obtained after the results from biochemical testing are available. Confirmatory diagnosis may require tissue or bone marrow biopsy in specific cases.

\section{Acknowledgement}

The authors would like to thank Dr. Hale Toklu and Ms. Megan Hubbard for their contributions.

\section{References}

1. Berg P, McCallum R. Dumping Syndrome: A Review of the Current Concepts of Pathophysiology, Diagnosis, and Treatment. Dig Dis Sci 2016;61(1):11-18.

2. van Beek AP, Emous M, Laville M, Tack J. Dumping syndrome after esophageal, gastric or bariatric surgery: pathophysiology, diagnosis, and management. Obes Rev 2017;18(1):68-85.

3. Sirinek KR, O’Dorisio TM, Howe B, et al. Neurotensin, vasoactive intestinal peptide, and Roux-en-Y gastrojejunostomy. Their role in the dumping syndrome. Arch Surg 1985;120(5):605-609.

4. Takahashi H, Shida D, Tagawa K, Suzuki T. Hemodynamics of mesenteric traction syndrome measured by FloTrac sensor. J Clin Anesth 2016;30:46-50.

5. Avgerinos DV, Theoharides TC. Mesenteric traction syndrome or gut in distress. Int J Immunopathol Pharmacol 2005;18(2):195-199.

6. Tan J, Berg M. Rosacea: Current state of epidemiology. J Am Acad Dermatol 2013;69(6)(Suppl 1):S27-S35.

7. Egeberg A, Weinstock LB, Thyssen EP, et al. Rosacea and gastrointestinal disorders: a population-based cohort study. $\mathrm{Br}$ J Dermatol 2017;176(1):100-106.

8. Crawford GH, Pelle MT, James WD. Rosacea: I. Etiology, pathogenesis, and subtype classification. J Am Acad Dermatol 2004;51(3):327-341; quiz 342-344.

9. Wollina U. Recent advances in the understanding and management of rosacea. F1000Prime Rep 2014;6:50.

10. Drummond PD, Su D. Endothelial and axon reflex vasodilatation to acetylcholine in rosacea-affected skin. Arch Dermatol Res 2012;304(2):133-137.

11. Dayan SH, Pritzker RN, Arkins JP. A new treatment regimen for rosacea: onabotulinumtoxinA. J Drugs Dermatol 2012;11(12):e76-e79.

12. Schwab VD, Sulk M, Seeliger S, et al. Neurovascular and neuroimmune aspects in the pathophysiology of rosacea. $\mathrm{J}$ Investig Dermatol Symp Proc 2011;15(1):53-62.

13. Two AM, Wu W, Gallo RL, et al. Rosacea: part 1. Introduction, categorization, histology, pathogenesis, and risk factors. J Am Acad Dermatol 2015;72(5):749-758, quiz 759-760.

14. Jørgensen A-HR, Egeberg A, Gideonsson R, Weinstock LB, Thyssen EP, Thyssen JP. Rosacea is associated with Helicobacter pylori: a systematic review and meta-analysis. J Eur Acad Dermatol Venereol 2017;31(12):2010-2015.

15. Spoendlin J, Karatas G, Furlano RI, et al. Rosacea in patients with ulcerative colitis and Crohns Disease: A populationbased case-control study. Inflamm Bowel Dis 2016;22(3):680687.

16. Abokwidir M, Feldman SR. Rosacea Management. Skin Appendage Disord 2016;2(1-2):26-34.
17. Shanler SD, Ondo AL. Successful treatment of the erythema and flushing of rosacea using a topically applied selective alpha1adrenergic receptor agonist, oxymetazoline. Arch Dermatol 2007;143(11):1369-1371.

18. De Leo S, Lee SY, Braverman LE. Hyperthyroidism. Lancet 2016;388(10047):906-918.

19. Chiha M, Samarasinghe S, Kabaker AS. Thyroid Storm: an updated review. J Intensive Care Med 2015;30(3):131-140.

20. Hambleton C, Buell J, Saggi B, Balart L, Shores NJ, Kandil E. Thyroid storm complicated by fulminant hepatic failure: case report and literature review. Ann Otol Rhinol Laryngol 2013;122(11):679-682.

21. Izikson L, English JC III, Zirwas MJ. The flushing patient: Differential diagnosis, workup, and treatment. J Am Acad Dermatol 2006;55(2):193-208.

22. Kemp SF, Lockey RF. Anaphylaxis: A review of causes and mechanisms. J Allergy Clin Immunol 2002;110(3):341-348.

23. Mali S, Jambure R. Anaphyllaxis management: Current concepts. Anesth Essays Res 2012;6(2):115-123.

24. Gnanavel S, Robert RS. Diagnostic and statistical manual of mental disorders, fifth edition, and the impact of events scalerevised. Chest 2013;144(6):1974.

25. Diagnostic and Statistical Manual of Mental Disorders. 5th edition ed. Washington, DC: American Psychiatric Association; 2013.

26. Kessler RC, Chiu WT, Jin R, Ruscio AM, Shear K, Walters EE. The epidemiology of panic attacks, panic disorder, and agoraphobia in the National Comorbidity Survey Replication. Arch Gen Psychiatry 2006;63(4):415-424.

27. Davies SJC, Jackson PR, Lewis G, Hood SD, Nutt DJ, Potokar JP. Is the association of hypertension and panic disorder explained by clustering of autonomic panic symptoms in hypertensive patients? J Affect Disord 2008;111(2-3):344-350.

28. Goodwin RD, Cowles RA, Galea S, Jacobi F. Gastritis and mental disorders. J Psychiatr Res 2013;47(1):128-132.

29. Lydiard RB, Greenwald S, Weissman MM, Johnson J, Drossman DA, Ballenger JC. Panic disorder and gastrointestinal symptoms: findings from the NIMH Epidemiologic Catchment Area project. Am J Psychiatry 1994;151(1):64-70.

30. Maunder RG. Panic disorder associated with gastrointestinal disease: Review and hypotheses. J Psychosom Res 1998;44(1):91-105.

31. Singh P, Agnihotri A, Pathak MK, et al. Psychiatric, somatic and other functional gastrointestinal disorders in patients with irritable bowel syndrome at a tertiary care center. J Neurogastroenterol Motil 2012;18(3):324-331.

32. de Montigny C. Cholecystokinin tetrapeptide induces panic-like attacks in healthy volunteers. Preliminary findings. Arch Gen Psychiatry 1989;46(6):511-517.

33. Fertleman CR, Baker MD, Parker KA, et al. SCN9A mutations in paroxysmal extreme pain disorder: allelic variants underlie distinct channel defects and phenotypes. Neuron 2006;52(5):767-774.

34. Fertleman CR, Ferrie CD, Aicardi J, et al. Paroxysmal extreme pain disorder (previously familial rectal pain syndrome). Neurology 2007;69(6):586-595.

35. Suter MR. What are the treatment options for paroxysmal extreme pain disorder? Pain Manage 2015;5(4):229-232.

36. Vetter I, Deuis JR, Mueller A, et al. Na V 1.7 as a pain target From gene to pharmacology. Pharmacol Ther 2017;172:73100.

37. Dib-Hajj SD, Estacion M, Jarecki BW, et al. Paroxysmal extreme pain disorder M1627K mutation in human Nav1.7 renders DRG neurons hyperexcitable. Mol Pain 2008;4:37.

38. Darbar A, Bilolikar A. Case reports: anesthesia for a patient with paroxysmal extreme pain disorder. Anesth Analg 2010;110(6):1719-1720. 
39. Cannon A, Kurklinsky S, Guthrie KJ, et al. Advanced Genetic Testing Comes to the Pain Clinic to Make a Diagnosis of Paroxysmal Extreme Pain Disorder. Case Rep Neurol Med 2016;2016:9212369.

40. Deng M, Nedorost S. Facial flushing: an uncommon presentation of serotonin toxicity. Dermatitis 2009;20(5):296297.

41. Hair PI, Curran MP, Keam SJ. Tramadol extended-release tablets. Drugs 2006;66(15):2017-2027; discussion 2028-2030.

42. Curran CF. Doxorubicin-associated facial flushing. Arch Dermatol 1992;128(10):1408.

43. Quertemont E. Genetic polymorphism in ethanol metabolism: acetaldehyde contribution to alcohol abuse and alcoholism. Mol Psychiatry 2004;9(6):570-581.

44. Sticherling M, Brasch J. Alcohol: intolerance syndromes, urticarial and anaphylactoid reactions. Clin Dermatol 1999; 17(4):417-422

45. Becker K, Southwick K, Reardon J, Berg R, MacCormack JN Histamine poisoning associated with eating tuna burgers. JAMA 2001;285(10):1327-1330.

46. Ferran M, Yébenes M. Flushing associated with scombroid fish poisoning. Dermatol Online J 2006;12(6):15.

47. Freeman M. Reconsidering the effects of monosodium glutamate: A literature review. J Am Acad Nurse Pract 2006;18(10):482-486.

48. Lurie Y, Wasser SP, Taha M, et al. Mushroom poisoning from species of genus Inocybe (fiber head mushroom): a case series with exact species identification. Clin Toxicol 2009;47(6):562565.

49. Vally H, Misso NLA, Madan V. Clinical effects of sulphite additives. Clin Exp Allergy 2009;39(11):1643-1651.

50. Mosqueda-Garcia R. Adenosine as a therapeutic agent. Clin Invest Med 1992;15(5):445-455.

51. Sidi V, Arsos G, Papakonstantinou E, et al. Use of amifostine in the treatment of recurrent solid tumours in children. Hippokratia. 2007;11(1):25-29.

52. Aronson JK, ed. Meyler's side effects of drugs: The international encyclopedia of adverse drug reactions and interactions. 16th ed. Waltham, MA: Elsevier BV; 2016.

53. Vieth M, Montgomery E. Medication-associated gastrointestinal tract injury. Virchows Arch 2017;470(3):245-266.

54. Kulisevsky J, Pagonabarraga J. Tolerability and safety of ropinirole versus other dopamine agonists and levodopa in the treatment of Parkinson's disease: meta-analysis of randomized controlled trials. Drug Saf 2010;33(2):147-161.

55. Goldstein I, Tseng LJ, Creanga D, Stecher V, Kaminetsky JC. Efficacy and Safety of Sildenafil by Age in Men With Erectile Dysfunction. J Sex Med 2016;13(5):852-859.

\section{Author Affiliations}

Vaibhav Rastogi, MD*, $†$; Devina Singh, MD Mazza, MD\$; Dipendra Parajuli, MDף, Steven H Yale, $M D^{*}, \dagger$

*University of Central Florida College of Medicine/HCA Consortium Graduate Medical Education, North Florida Regional Medical Center, $6500 \mathrm{~W}$ Newberry Rd, Gainesville, FL 32605.

†University of Central Florida College of Medicine, 6850

Lake Nona Blvd, Orlando, FL 32827.

$\neq$ Feinstein Institute for Medical Research, 350 Community

Dr. Manhasset, NY 11030

\$Marshfield Clinic Research Institute, 1000 North Oak

Avenue, Marshfield, WI 54449.
IUniversity of Louisville, Department of Medicine, Gastroenterology, Hepatology and Nutrition. Director, Fellowship Training Program, Director, Medical Procedure Unit Louisville VAMC 401 East Chestnut Street, Louisville, KY 40202 